\title{
LOCAUZACIÓN DEL CANCER DE PRÓSTATA EN LA GLÁNDULA CENTRAL MEDIANTE ESPECTROSCOPIA DE RESONANCIA MAGNÉTICA ENDORECTAL
}

\author{
Josep Comet Battle, Joan Carles Vilanova Busquets², Albert M aroto G enover ${ }^{3}$, Joan Areal \\ Calama ${ }^{1}, M$ argarita $O$ sorio Fernández ${ }^{3}$, Eugeni López Bonet ${ }^{4}, N$ arcis Torrent $Q$ uer, Miquel \\ O rdis Dalmau, Josep M aría Saladié Roig ${ }^{1}$ y Carles Barceló Vidal5.
}

\begin{abstract}
Agencia de Evaluación de Tecnología para la Investigación M édica (AATRM). Servicio de Urología. Hospital Universitari Dr. Josep Trueta. G irona.

Servicio de Urología ${ }^{1}$. Hospital G ermans Trias i Pujol. Badalona.

Ressonància Magnètica Girona². Girona.

Servicio de Radiología3. Hospital Universitari Dr. Josep Trueta. G irona.

Servicio de Anatomía Patológica ${ }^{4}$. Hospital Universitari Dr. Josep Trueta. G irona. Facultad de M atemática Aplicada ${ }^{5}$. Universitat de G irona UdG. G irona. España.
\end{abstract}

\begin{abstract}
Resumen.- O BJETIVO: La espectroscopia de resonancia magnética endorectal (E-RME) es una nueva técnica de imagen que permite una evaluación más acurada y fiable de la localización y estadiaje del cáncer de próstata $(\mathrm{C}$ aP) que la resonancia magnética endorectal sola. La combinación de la RME y la E-RME ha conseguido recientemente mejorías técnicas que han permitido aumentar la fiabilidad en la detección del CaP. N uestro grupo está a ctualmente trabajando con la E-RM E en la detección del CaP, en colaboración con la Agencia de Evaluación de Tecnología para la Investigación M édica (AATRM), y en espera de resultados definitivos podemos avanzar que ésta técnica
\end{abstract}

Josep C omet Batlle

Servicio de Urología

Hospital Universitari Dr. J. Trueta

C tra $\mathrm{N}$ acional II, $\mathrm{s} / \mathrm{n}$

17007 G irona. (España)

e-mail: 28547jcb@comb.es

Trabajo recibido: 8 de agosto 2004 puede ser utilizada como una buena alternativa en la localización de $\mathrm{C}$ aP en pacientes con biopsias previas negativas pero en quienes persiste la sospecha de $C$ aP. M ÉTO DO S: Presentamos aquí una serie de 5 casos clínicos de pacientes controlados por elevación persistente del PSA y biopsias previas negativas. Realizamos biopsias por sextantes mediante ecografía transrectal a intervalos de 6 meses, después de determinar los valores de PSA. La RME y E-RME se realizó para intentar localizar el $\mathrm{CaP}$ y de este modo intentar minimizar el número de biopsias.

RESULTADO S: Todos los pacientes en esta serie presentaron una lesión de baja intensidad localizada en la hipointensidad normal de la glándula central, pero con una clara alteración metabólica en la espectroscopia que sugería la existencia de un $\mathrm{CaP}$, tal como se demostró posteriormente por biopsia.

CON CLUSIO NES: La E-RME es un método poco invasivo que ofrece la capacidad de detectar el CaP en la glándula central con mayor fiabilidad en pacientes seleccionados. La glándula central es una zona en la que el CaP se localiza con menor frecuencia, pero a menudo adopta la misma intensidad de señal que el tejido hiperplásico, y por tanto, resulta difícil de detectar por métodos puramente morfológicos. La E-RM E permite evaluar las alteraciones metabólicas en toda la glándula y aumentar así la fiabilidad en la detección del CaP, tanto en la glándula central como en la periférica. 
Palabras clave: Spectroscopy. Endorectal M RI. Prostate cancer. Staging. Detection.

Summary.- O BJEC TIVES: The endorectaI M $R$ spectroscopic imaging is a new imaging test which allows more accurate and reliable localization and staging of prostate cancer than simple endorectal M RI. The combination of spectroscopic $M R$ and $M R I$ has recently achieved technical improvements that increased reliability in the detection of prostate cancer. 0 ur group is now working in the detection of prostate cancer with the spectroscopic $M R$, in co-operation with the Agency for the Evaluation of Technology for M edical Research (Agencia de Evaluación de Tecnología para la Investigación M édica-AATRM ); although we are waiting for definitive results, we can advance that this technique may be used as a good alternative for localization of prosta te cancer in patients with previous negative biopsies in whom the suspicion of prosta te cancer persists.

METHODS: We present a series of 5 patients under control for permanent elevation of PSA with previous negative biopsies. W e were performing ultrasound guided sextant biopsies every 6 months, after blood test for $P S A$. Endorectal MRI and spectroscopic MRI were performed to try to localize the prostate cancer so diminishing the number of biopsies.

RESULTS: All patients in the series had a low intensity lesion within the normal low intensity of the central gland, with an obvious spectroscopic metabolic abnorma lity suggesting the existence of prosta te cancer, as it was then demonstrated by biopsy.

CONCLUSIONS: The endorectal MR spectroscopic imaging is a non invasive method which offers the ability to detect prosta te cancer within the centra I gland with a higher reliability in selected patients. The central gland is an area in which prostate cancer is less commonly localized, but it often shows the same signal intensity than hyperplastic tissue, so that it is difficult to be detected by purely morphological methods. Endorectal M R spectroscopic imaging allows evaluating the metabolic disturbances in the whole gland, increasing the reliability of detection of prostate cancer both in the central and peripherical glands.

Keywords: Spectroscopy. Endorectal M RI. Prostate cancer. Staging. Detection.

\section{INTRODUCCIÓN}

Ya en el año 1964, cuando Cooper y Farid presentaron sus trabajos sobre los contenidos de citrato en la próstata establecieron que "en la próstata humana se pueden producir cambios bioquímicos mesurables durante su transformación maligna, mucho antes de que el diagnóstico histológico pueda ser realizado por el patólogo. Si esta premisa puede ser comprobada, la detección bioquímica de la malignidad en la próstata podrá ser posible en los estadios precoces, formativos del carcinoma de próstata, cuando no hay signos clínicos evidentes" $(1,2)$. Desde entonces, varios estudios han acumulado evidencia sobre la relación existente entre las variaciones del citrato prostático y otros metabolitos y la existencia o no de tumor en el tejido. Esta relación puede variar de forma evidente dependiendo de la localización central o periférica del tejido analizado, puesto que la cantidad de citrato es también variable en las diferentes regiones de la glándula (3).

En 1954, Cooper e Imfeld establecen que los niveles de citrato en el tejido prostático benigno (normal e hiperplásico) eran significativamente más elevados que los niveles de citrato en el tejido maligno (4). Y posteriormente Marberger (5), en 1962 describe que se produce una dramática disminución de los niveles de citrato en el tejido metastático de CaP respecto al tejido normal. A simismo, estos cambios son más pronunciados a nivel glandular que a nivel estromal, puesto que el estroma prostático contiene poco citrato (6).

A unque los niveles de citrato en el CaP son significativamente inferiores al tejido normal o hiperplásico, existe una variabilidad en las concentraciones del mismo debido a la composición del tejido a nalizado, y para minimizar estas variaciones las concentraciones de citrato son habitualmente referenciadas respecto a la colina (cociente colina/ citrato) 0 colina+creatina (cociente colina+creatina/ citrato), ya que se ha determinado que las concentraciones de colina aumentan en el tejido maligno, y ello conduce a un aumento de la discriminación metabólica del cáncer respecto a zonas periféricas normales $(7,8)$.

A ctualmente existe la tecnología para medir las concentraciones de citrato y otros metabolitos en la próstata entera, mediante la utilización de la espectroscopia de resonancia magnética endorectal (E-RME). 


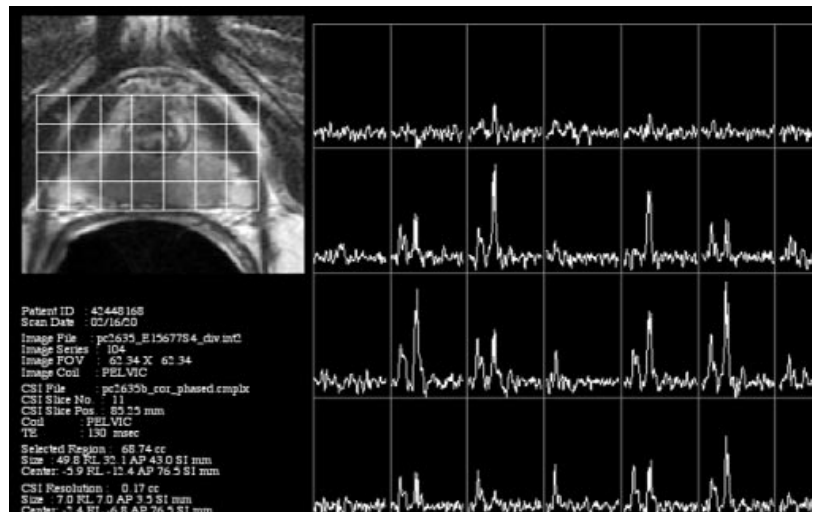

FIG URA 1. Espectros contiguos sobreimpresos al estudio morfológico prostático.

La E-RME reproduce una serie de espectros contiguos en 1, 2 y 3 dimensiones por cada volumen de tejido de 0,24 cc. aproximadamente, y esta información metabólica se sobrepone a la imagen anatómica-morfológica. (Figura 1).

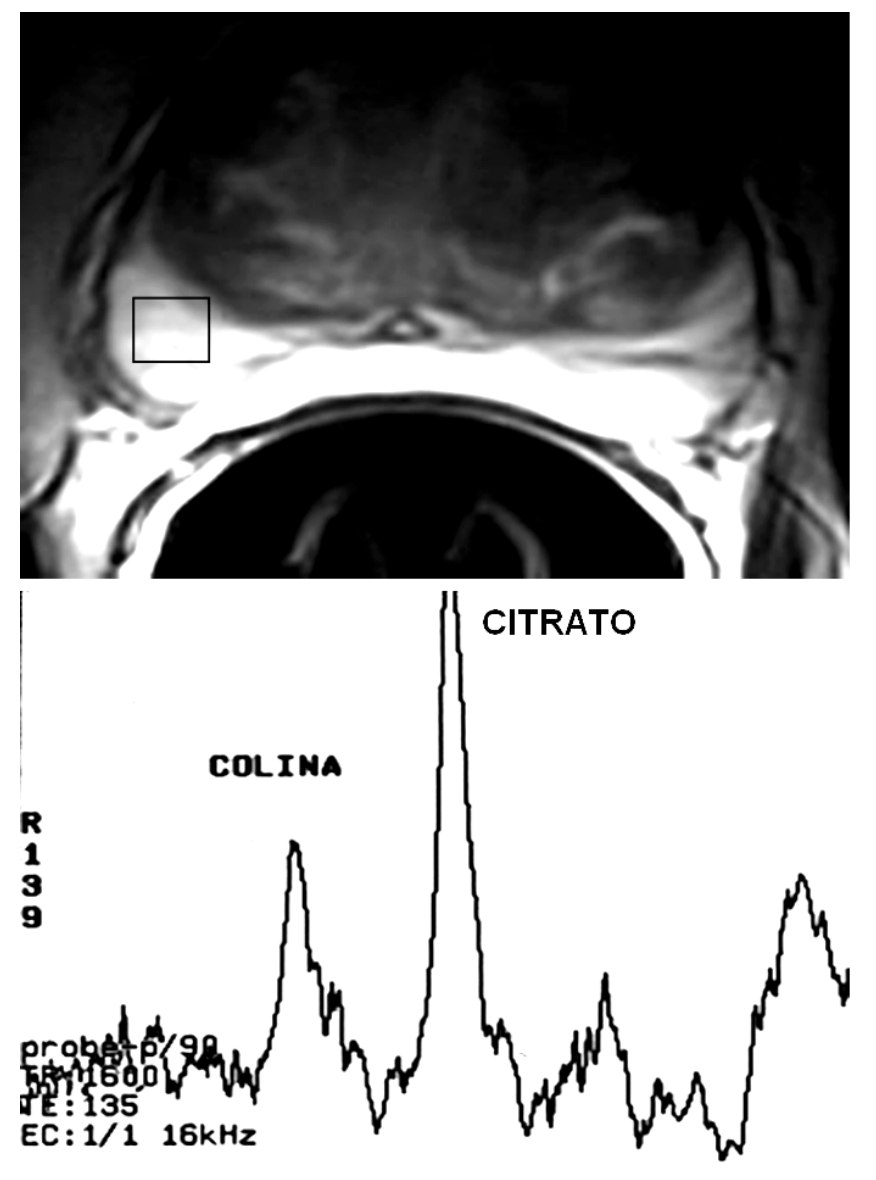

FIG URA 2. Patrón normal
Cada uno de estos espectros tiene una configuración especifica, y por tanto un cociente colina+creatina/citrato diferente, que será más 0 menos sugestivo de neoplasia en función del valor. Se define como posible cáncer un cociente colina+creatina/ citrato superior a 2 DS por encima del normal $(>0,75)$, y probable cáncer cuando se produce una elevación del cociente 3 DS por encima del normal $(>0,86)$. Los voxels con un cociente inferior a 0,75 se consideran tejido normal.

El patrón espectroscópico normal de la glándula periférica prostática se caracteriza por unos niveles elevados de citrato y bajos niveles de colina en el tejido, tal como se muestra en el esquema (Figura 2), curva que se invierte en el caso de neoplasia.

\section{CASOS CÚNICOS}

\section{Caso clínico 1}

Paciente de 65 a. de edad, que presentaba una elevación progresiva de las cifras de PSA desde $6 \mathrm{ng} / \mathrm{ml}$
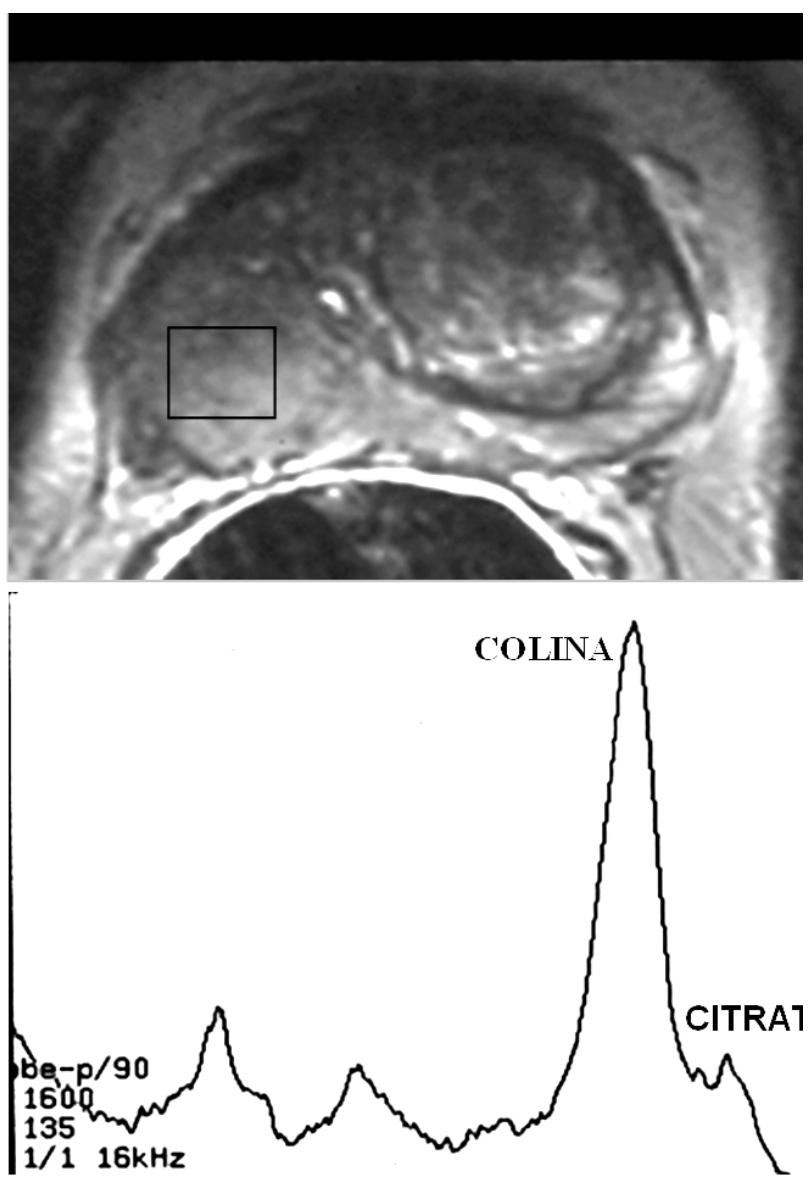

$N$ eoplasia de próstata 

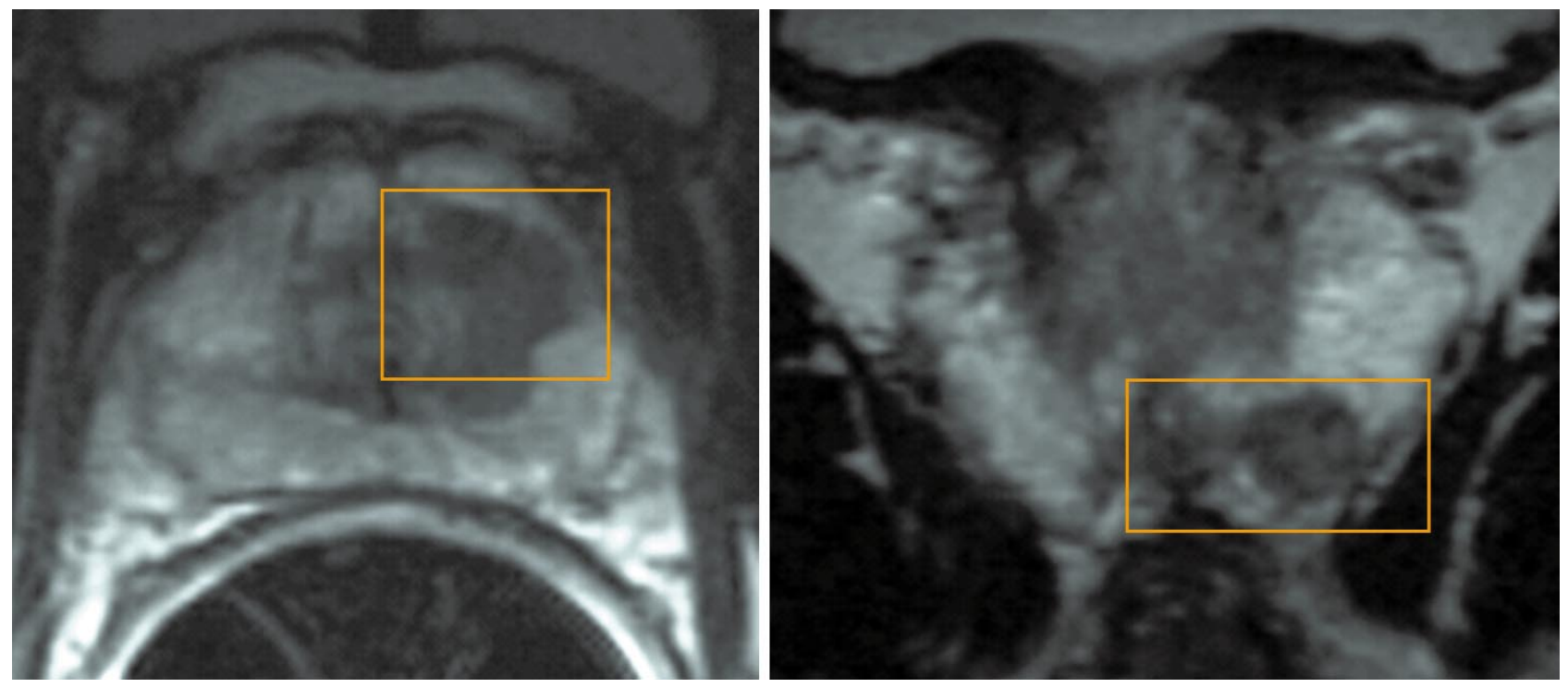

FIG URA 3. Imagen hipointensa en ápex de zona periférica y central del $D$

a $12 \mathrm{ng} / \mathrm{ml}$ en el último año. Dicho paciente había sido sometido un año antes a una biopsia transrectal de próstata ecodirigida por sextantes que fue negativa.

Se decide, ante la elevación del PSA, realizar una E-RME previa a la 2a biopsia transrectal, en la que se objetiva una lesión hipointensa que abarca zona central i periférica del ápex del lóbulo $D$ prostático (Figura 3). El estudio espectroscópico de ésta lesión mostró una curva de colina discretamente más elevada que la curva de citrato, con un índice de $\mathrm{Ch}-\mathrm{Cr} / \mathrm{Ci}$ $>0,80$ (Figura 4), que sugería la presencia de lesión neoplásica, por lo que se biopsia dicha zona por ecografía transrectal. La AP de la biopsia confirmó la presencia de un ADK próstata Gleason 6.

\section{Caso clínico 2.}

Se trata de un paciente de $60 \mathrm{a}$. de edad, con una elevación del PSA de $7 \mathrm{ng} / \mathrm{ml}$, posteriormente $15 \mathrm{ng} / \mathrm{ml}$ y

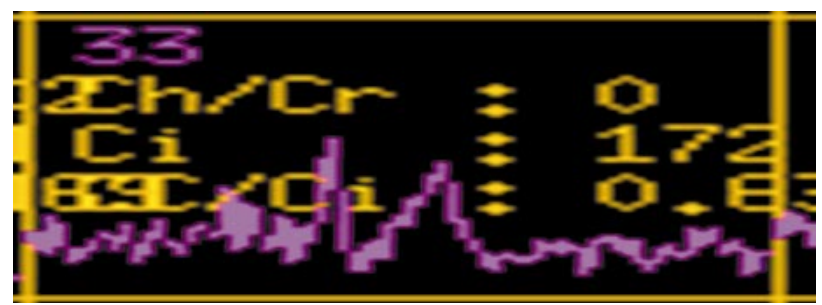

FIG URA 4. Espectroscopia con cociente sugestivo de neoplasia.
$32 \mathrm{ng} / \mathrm{ml}$ meses más tarde. El paciente había sido sometido previamente a 3 biopsias prostáticas por sextantes, por via transrectal y ecodirigidas. Se decidió hacer en este paciente una E-RME previa a la última biopsia y nos hallamos con un diagnóstico espectroscópico sugerente de neoplasia de próstata, por lo que la sospecha inicial se produjo por la espectroscopia. La resonancia magnética endorectal no mostraba en este paciente ninguna alteración sugestiva de neoplasia desde el punto de vista morfológica (Figura 5). Sin embargo al realizar la espectroscopia de ambas zonas centrales, se objetivó en el lado derecho una curva espectroscópica de aspecto normal, con una elevación marcada del citrato a 2,5 ppm (partes por millón del espectro) y una curva menos marcada de colina-creatina (curva de doble pico situada entre 3,0 y 3,2 ppm en el espectro), y en el lado izquierdo, aleatoriamente se objetivó una zona con una elevación de colina más marcada que la correspondiente curva de citrato, sugerente por tanto de neoplasia, que se confirmó por biopsia posteriormente, mediante punción de la zona central de ambos lóbulos

(Figura 6).

\section{Caso clínico 3.}

Paciente de 69 a. de edad con un PSA en el momento de la consulta de $22 \mathrm{ng} / \mathrm{ml}$. El paciente tenía una biopsia previa por sextantes negativa. Se practicó una E-RME que mostró inicialmente un nódulo situado en la base prostática del lóbulo izquierdo, morfológicamente sugestivo de hiperplasia nodular (Figura 7), pero el 

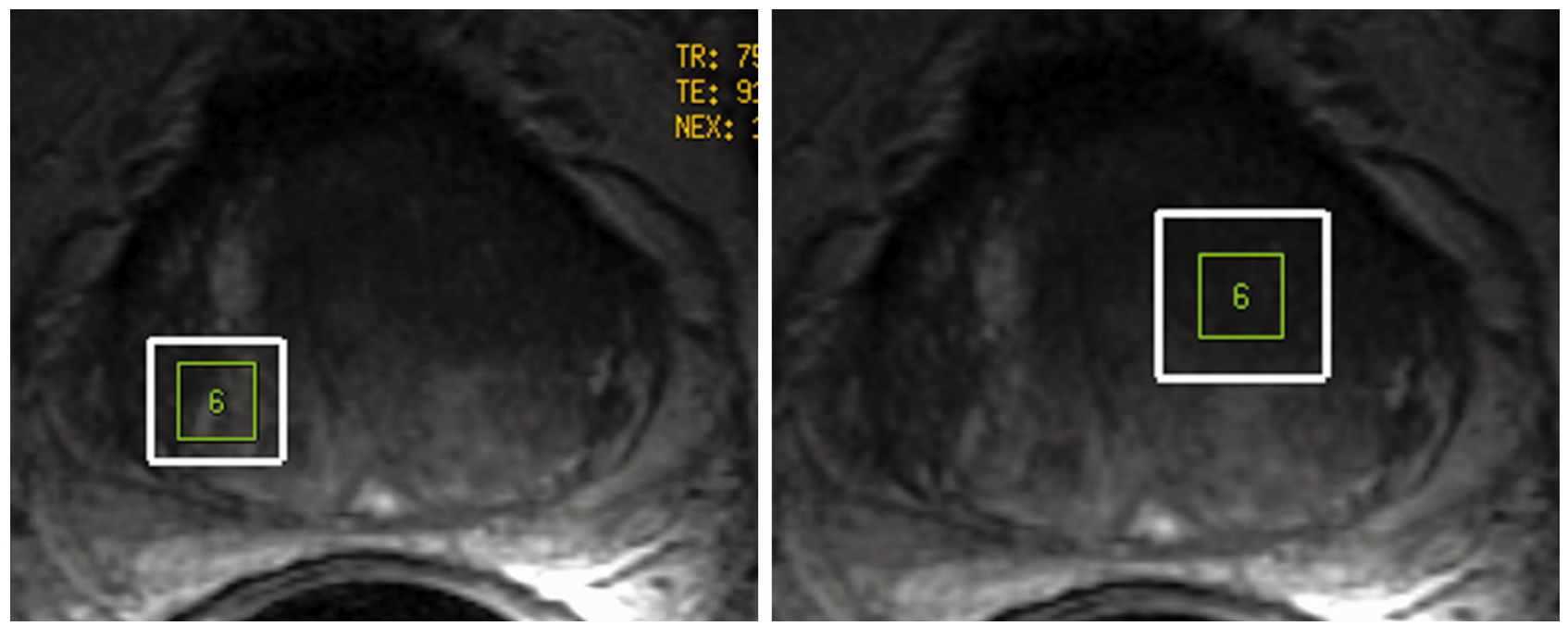

FIG URA 5. Resonancia endorectal sin imágenes sospechosas de neoplasia

estudio espectroscópico mostró una alteración metabólica de este nódulo, con una importante elevación de la colina y una disminución del citrato, sugerente de neoplasia (Figura 8).

\section{Caso clínico 4.}

Paciente de 70 a. con PSA de $30 \mathrm{ng} / \mathrm{ml}$. Los valores previos de PSA eran de 12 y $19 \mathrm{ng} / \mathrm{ml}$ respectivamente. El paciente había sido sometido a 2 biopsias prostáticas previas, la 1 a por sextantes y la $2 \underline{a}$ mediante 8 muestras, puesto que por entonces ya habíamos empezado en nuestro servicio a tomar 8 muestras en los casos de rebiopsia.

Al realizar la E-RME se objetivó una extensa área hipointensa central (Figura 9), que por espectroscopia mostraba en algunas zonas unos índices sugestivos de neoplasia, tal como se muestra en la figura (Figura $10)$, sobre todo en los voxels $29,30,31$ y 32 . En estos voxels se observan curvas de colina elevadas con respecto a las de citrato, lo cual sugiere la presencia de neoplasia en esta zona. En dichos gráficos se observa también una 3 a curva, de banda ancha que habitualmente corresponde a la presencia de grasa.

Posteriormente la biopsia confirmó la presencia de un adenocarcimoma Gleason $7(3+4)$ en la zona central de la próstata.

\section{Caso clínico 5.}

Paciente de 62 años de edad con discretas molestias miccionales irritativas, tacto rectal no sugestivo de neo-
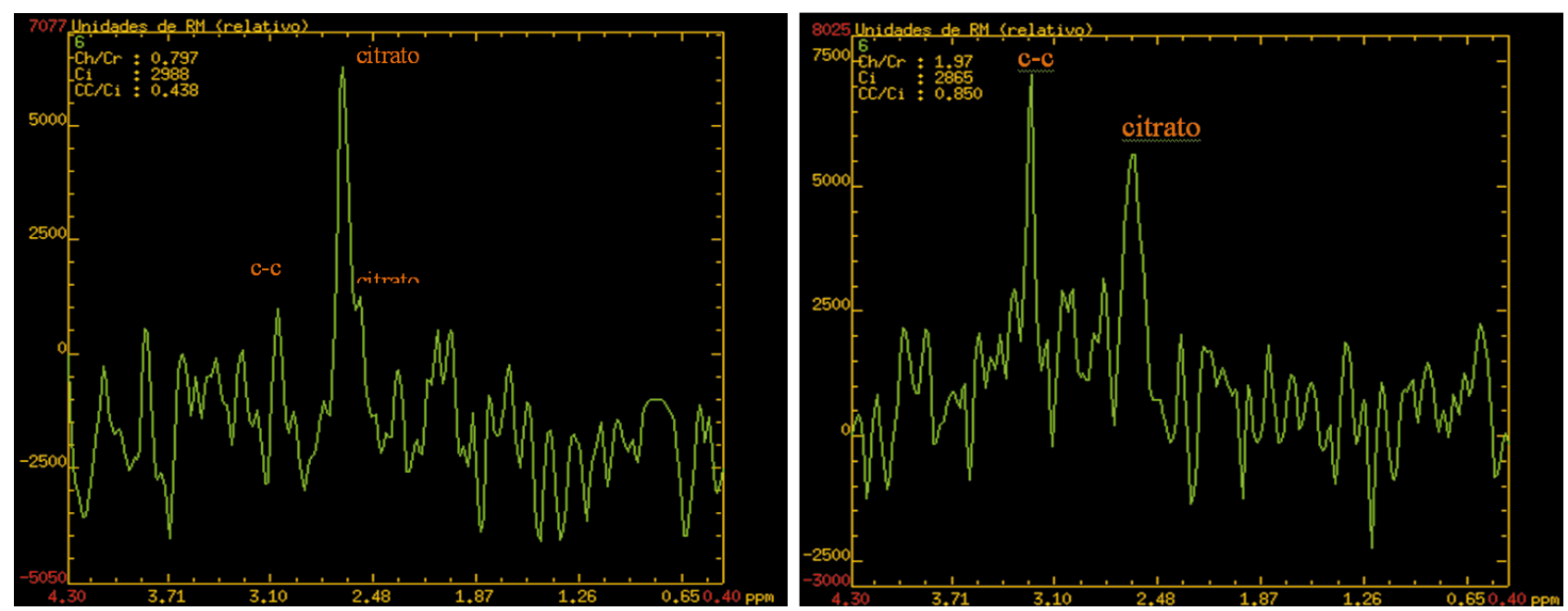

FIG URA 6: Espectroscopia correspondiente a las imágenes de Fig. 5 

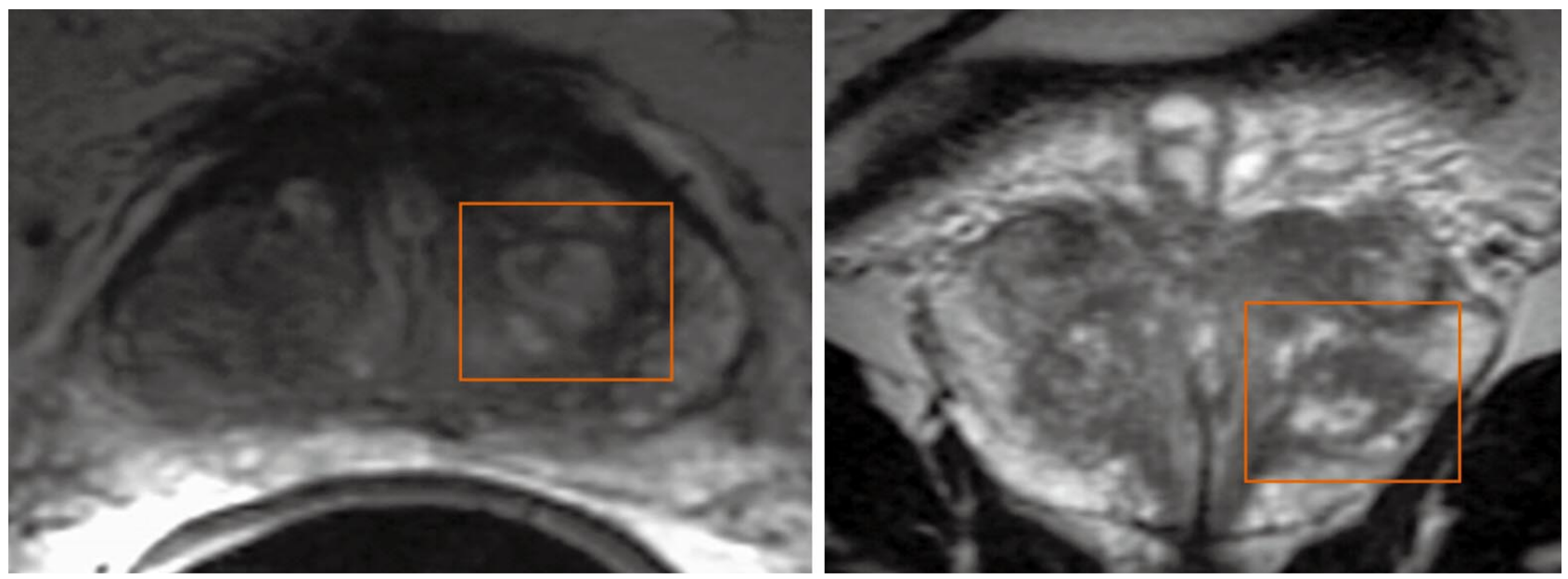

FIG URA 7. N ódulo sugestivo de hiperplasia nodular prostática

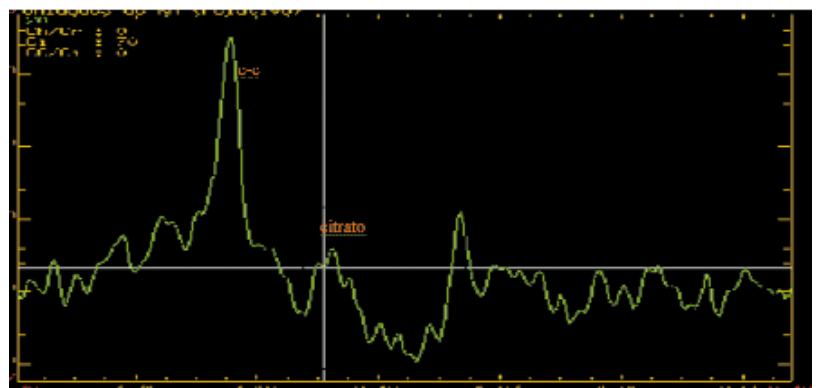

FIG URA 8. Espectroscopia sospechosa de neoplasia prostática

plasia. El valor de PSAt era $29,5 \mathrm{ng} / \mathrm{ml}$. Los valores previos de PSA total / PSA libre/ índice fueron de 14,2 / 0,80/ 5,6\% y $12,0 / 0,69 / 5,7 \%$ respectivamente.

El paciente había sido sometido a 5 biopsias previas

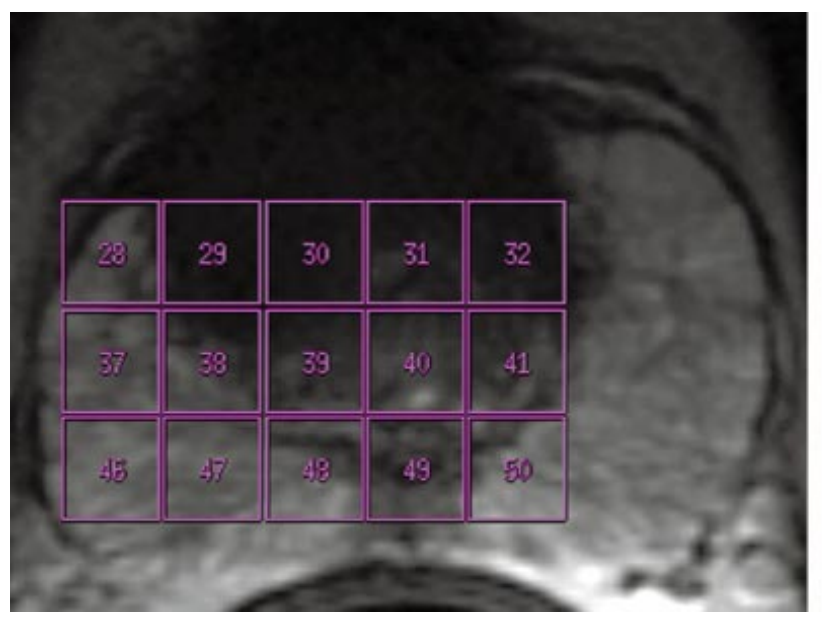

por sextantes, todas ellas negativas. Se practicó entonces una E-RME que mostró, en imágenes axiales y coronales, la presencia de una amplia zona hipo intensa a nivel del ápex de la zona central prostática (Figura 11), que en la espectroscopia presentaba áreas sugestivas de neoplasia. A pesar de tener unos índices elevados de creatina-colina/ citrato sugestivos de neoplasia, los voxels 36 y 45 son poco valorables puesto que corresponden a la zona de uretra prostática. En los voxels $46,52,53$ y 55 se observan unas curvas elevadas de citrato con unos picos de colina menos acentuados, y unos índices poco sugestivos de neoplasia $(<0,75)$. En el resto de gráficos, se observa una disminución generalizada de los metabolitos (Figura 12), con unos índices sospechosos de neoplasia, que se confirmó mediante biopsia de dicha zona.

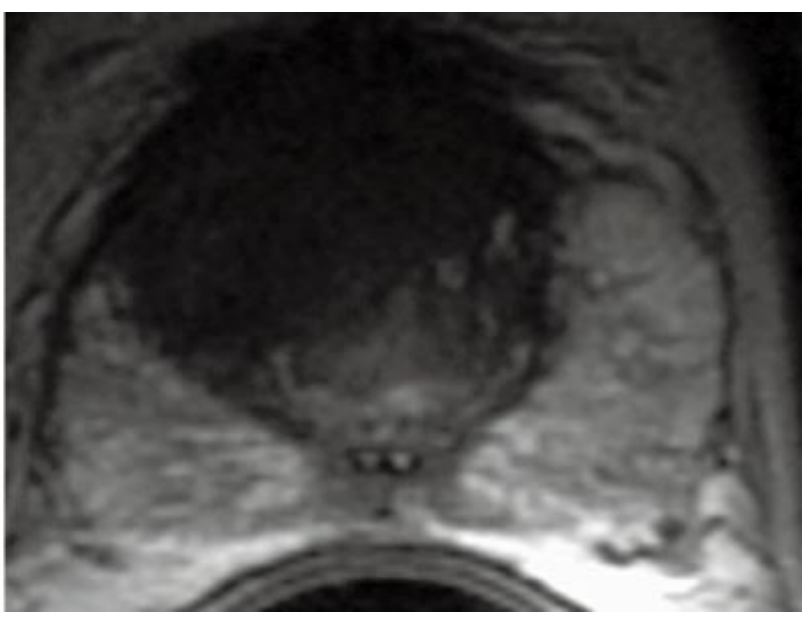

FIG URA 9. Área hipo intensa en la zona central de la próstata por resonancia 


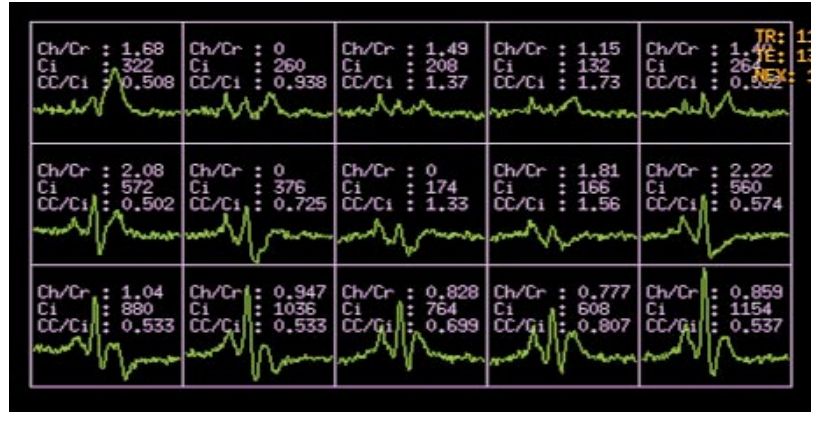

FIG URA 10. Espectros sugestivos de neoplasia $(29,30,31$ y 32$)$.

\section{DISCUSIÓN}

A ctualmente se acepta que la RM con bobina endorectal constituye el método no invasivo más fiable para identificar el estadio localmente avanzado en el cáncer de próstata, especialmente la afectación de vesículas seminales, puesto que proporciona imágenes de gran resolución de la próstata y de la infiltración del tejido circundante (10)

Sin embargo, en estudios de nuestro grupo de trabajo publicados anteriormente (11) así como otras publicaciones incluso previas que reforzaban la misma a firmación, se objetiva que la sensibilidad y especificidad de la RME oscila entre $60-80 \%$, según las publicaciones, y que la RME sola no permite predecir de forma fiable las biopsias positivas en pacientes con PSA de $4-10 \mathrm{ng} / \mathrm{ml} 0$ de $10-20 \mathrm{ng} / \mathrm{ml}$ respectivamente. Concluíamos también en este trabajo que los pacientes con resonancia negativa no debían dejar de biopsiarse puesto que nos encontrábamos un número no despreciable de ellos con falsos negativos. De todas maneras, se observa que la resonancia proporciona un importante elemento de apoyo en los pacientes en que existe una discordancia clínico-patológica, con persistencia de la elevación del PSA pero biopsias negativas. En estos pacientes observamos que la resonancia proporcionaba a menudo la información necesaria para la detección del tumor. En este sentido, se publicó posteriormente un trabajo (12) en el que se analizaba el valor de la resonancia en el diagnóstico precoz de pacientes controlados por elevación del PSA 0 alteración del TR, y se observó que el valor negativo de una resonancia endorectal en pacientes con biopsias previas negativas reducía notablemente la probabilidad de obtener una biopsia positiva.

Simultáneamente a todos estos hallazgos, fue perfeccionando progresivamente la tecnología de espectroscopia de resonancia y ya en 1999-2000 se publicaron diversos trabajos que concluían que la $\mathrm{E}$ RME permitía aumentar en general la fiabilidad en la detección del CaP y que además reducía la variabilidad ínter observador existente en la RM endorectal sola (13). Se observó que esta técnica permitía mejorar la predicción de la afectación extracapsular (13), mejoraba la predicción del volumen tumoral de forma significativa (14) e incluso tenía una fiabilidad similar a la biopsia cuando se comparaban ambas técnicas, siendo en algunas localizaciones de la próstata más fiable la E-RME que la biopsia (15).

En general pues, podemos decir que el análisis bioquímico de los niveles de citra to y otros metabo-
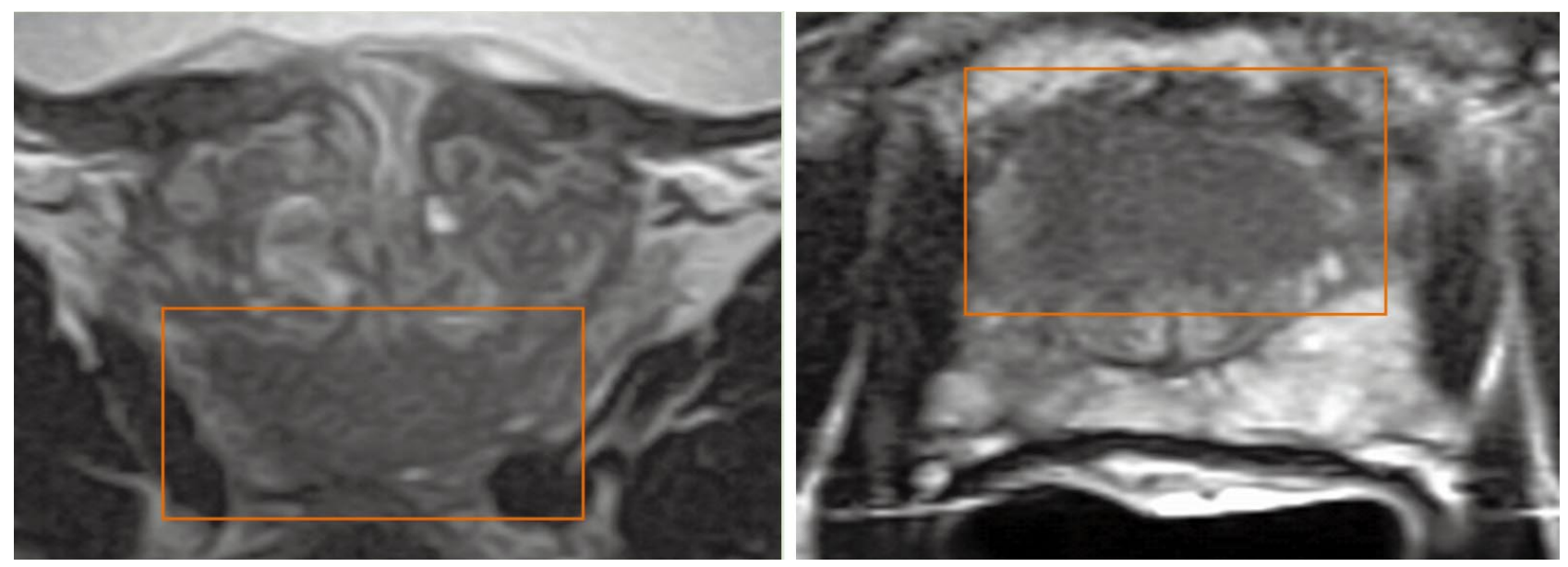

FIG URA 11. Imagen axial y coronal que muestra área hipointensa central 

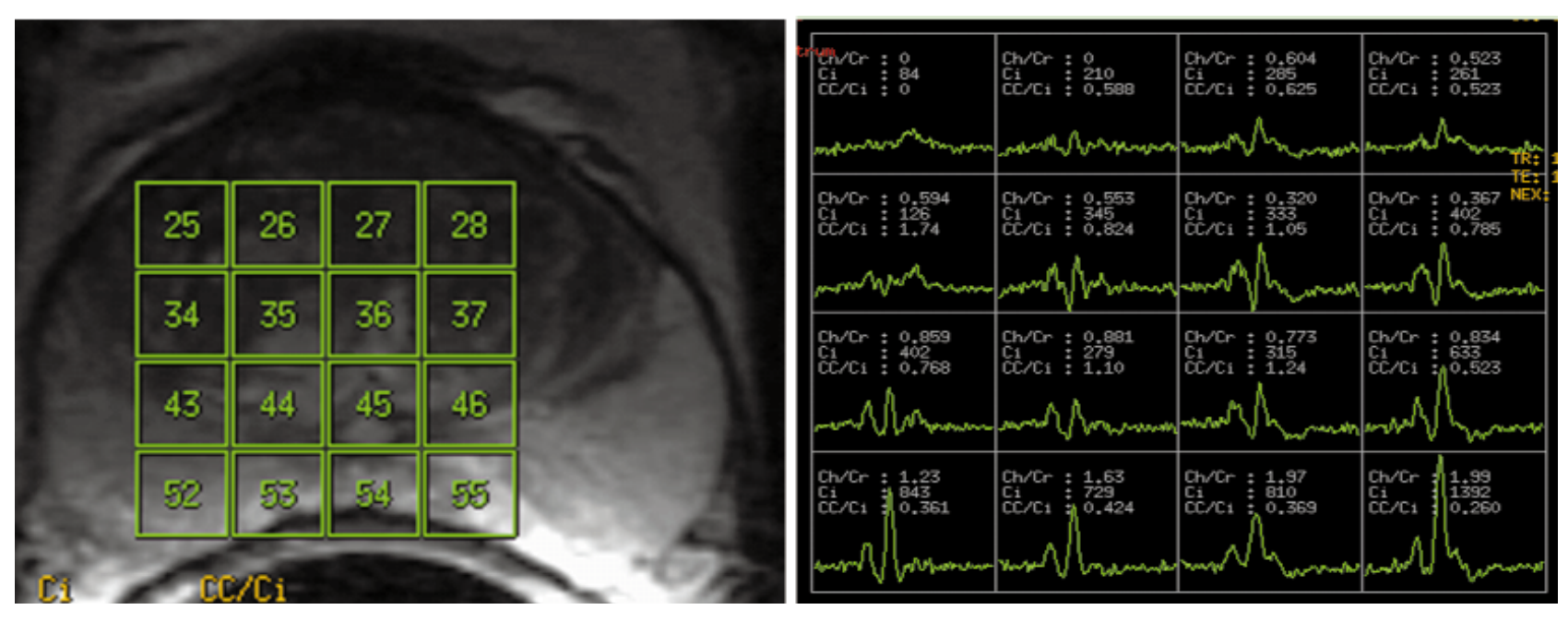

FIG URA 12. Espectro sugestiva de neoplasia

litos en la próstata proporciona un aumento importante de la especificidad en la detección del CaP con respecto a la RME sola (con numerosos falsos positivos en casos de hemorragia post-biopsia, prostatitis, tratamientos previos... ), y globa Imente mejora la fiabilidad en la localización de esta patología (9). Por tanto la ERME se perfila como un método no invasivo prometedor para la detección, estadiaje y seguimiento del CaP. En nuestro grupo estamos actualmente trabajando con esta técnica y evaluando qué valor aporta en el diagnóstico precoz de los pacientes en estudio por elevación del PSA y/ 0 alteración del TR, y simultáneamente evaluamos pacientes multibiopsiados con biopsias previas negativas. Los resultados están todavía es fase de recogida pero se intuye que esta técnica aportará importantes datos en el diagnóstico del $\mathrm{CaP}$.

A medida que ha aumentado la aceptación de ésta técnica como instrumento de evaluación del CaP, ha aumentado también la variabilidad de los estudios relacionados con la técnica en sí. Hasta recientemente, la evaluación de la E-RME en el CaP se ha focalizado exclusivamente en la zona periférica (ZP), pero cabe recordar que en ella se localiza aproximadamente el $65 \%$ de tumores, estando el $30 \%$ restante localizado en la zona transicional (ZT) de la próstata. El estudio de esta última mediante técnicas de imagen (incluyendo Eco y RM) es a menudo muy difícil puesto que da origen a la hiperplasia benigna de próstata, que con frecuencia tiene una apariencia muy heterogénea. Cabe destacar un artículo reciente de Zaklan y cols. (16) en el que estudian si el patrón metabólico de los tumores detectados en la zona central tiene las mismas características que las alteraciones metabólicas detectadas en la zona periférica y que hasta hoy son la referencia para establecer el diagnóstico espectroscópico del CaP mediante E-RME. Es decir, el objetivo es determinar si los tumores de la ZP y de la ZT tienen las mismas características metabólicas. Llegan a la conclusión de que el perfil metabólico de los tumores localizados en la ZT es diferente del perfil de la ZT benigna, pero la amplia variabilidad de patrones en los tumores localizados en la ZT excluye el uso de un patrón concreto como referencia, tal como sucede en la zona periférica.

Este hecho tiene especial importancia para la detección del $\mathrm{CaP}$ en la ZT, puesto que por una parte, está ampliamente aceptado que debe excluirse la biopsia rutinaria inicial de la ZT por su bajo rendimiento (17), y reservarla para los casos de rebiopsias en pacientes con biopsias previas negativas. Pero por otra parte, a menudo nos hallamos con adenomas voluminosos que contienen tumores de relativo poco volumen y por tanto el éxito de localizar un tumor mediante punción sola puede reducirse notablemente. Por tanto, definir los patrones estándar de patología tumoral en la glándula central será de crucial importancia en el futuro reciente, puesto que la E-RME puede aportar una valiosa información en la evaluación de estos pacientes antes de la biopsia, tal y como hemos visto en los casos expuestos en este articulo. $Y$ en este sentido, recientemente se presentará una comunicación de nuestro grupo en que se evalúan las 
diferencias metabólicas de los tumores hallados mediante espectroscopia en la ZT y la ZP que puede aportar más información a estas incógnitas (18).

\section{CONCLUSIÓN}

La glándula central es una localización menos frecuente del $\mathrm{CaP}$, aproximadamente del orden del $20 \%$ de tumores se originan en esta situación. Sin embargo los tumores de localización central tienen la particularidad de que a menudo se presentan por resonancia endorectal con una intensidad muy similar a la del adenoma, y por tanto se hace difícil el diagnóstico morfológico en esta situación, especialmente en pacientes con adenomas muy voluminosos o heterogéneos.

La E-RME ofrece la posibilidad de evaluar la presencia de alteraciones metabólicas en toda la glándula prostática, y de esta manera aumentar la fiabilidad en la detección del CaP, tanto a nivel de la glándula central como de la glándula periférica.

En definitiva, esta técnica constituye un método poco invasivo que habrá que valorar en un futuro próximo para aumentar la detección de $\mathrm{CaP}$ en pacientes previamente biopsiados, con persistencia de la elevación del PSA, o de sospecha de neoplasia.

\section{BIBUOGRAFIA y LECTURAS \\ RECOMENDADAS (*lectura de interés y **lectura fundamental)}

1. COOPER, J.E.; FARID, I.: "The role of citric acid in the physiology of the prostate. A chromatographic study of citric acid cycle intermediates in benign and malignant prostatic tissue”. J. Surg. Res., 3: 112, 1963.

2. COOPER, J.E.; FARID, I.: "The role of citric acid in the phisiology of the prostate. Lactic/citrate ratios in benign and malignant prostatic homogenates as an index of prostatic malignancy". J. Urol., 92: 533, 1964.

**3. COSTELLO, L.C.; FRANKLIN, R.B.; NARAYAN, P.: "Citrate in the diagnosis of prostate cancer". The prostate, 38: 237, 1999.

4. COOPER, J.F.; IMFELD, H.: "The role of citric acid in the physiology of the prostate : a preliminary report". J. Urol., 81: 157, 1959.
5. MARBERGER, H.; MARBERGER, E.; MANN, T. y cols.: "Citric acid in human prostatic secretion and metastatazing cancer of prostate gland". Br. Med. 1: 835, 1962.

6. COSTELLO, L.C.; LITTLETON, G.; FRANKLIN, R.B.: "Regulation of citrate related metabolism in normal and neoplastic prostate. En: Sharma RK, Criss WE, editors. Endocrine control in neoplasia". New York: Raven Press, 1978.

7. KURHANEWICZ, J.; VIGNERON, D.B.; NELSON, S.J. y cols.: "Citrate as an in vivo marker to discriminate prostate cancer from benign prostatic hyperplasia and normal prostate peripheral zone: detection via localized proton spectroscopy". Urology, 45: 459, 1995.

8. HEERSCHAP, A.; JAGER, J.G.; VAN DER GRAFF, M. y cols.: "In vivo proton MR spectroscopy reveals altered metabolite content in malignant prostate tissue". Anticancer Res., 17: 1455, 1997.

**9. SCHEIDLER, J.; HRICAK, H.; VIGNERON, D.B. y cols.: "Prostate cancer : localization with three-dimensional proton MR spectroscopic imaging". Clinicopathologic study. Radiology, 213: 473, 1999.

10. EAU Guidelines. Prostate cancer. Staging.

*11. VILANOVA, J.C.; COMET, J.; CAPDEVILA, A. y cols.: "The value of endorectal MR imaging to predict positive biopsies in clinically intermediate-risk prostate cancer patients". Eur. Radiol., 11: 229, 2001.

*12. COMET, J.; VILANOVA, J.C.; SALADIÉ, J.M. y cols.: "The value of endorectal MRI in the early diagnosis of prostate cancer". European Urology, 44: 201, 2003.

13. YU, K.K.; SCHEIDLER, J.; HRICAK, H. y cols.: "Prostate cancer: prediction of extracapsular extension with endorectal MR imaging and three.dimensional proton MR spectroscopic imaging". Radiology, 213: 481, 1999.

14. COAKLEY, F.V.; KURHANEWICZ, J.; LU, Y. y cols.: "Prostate cancer tumor volume: measurement with endorectal MR and MR spectroscopic imaging". Radiology, 223: 91, 2002.

15. WEFER, A.E.; HRICAK, H.; VIGNERON, D.B. y cols.: "Sextant localization of prostate cancer: comparison of sextant biopsy, magnetic resonance imaging and magnetic resonance spectroscopic imaging with step section histology". J. Urol., 164: 405, 2000.

**16. ZAKLAN, K.L.; EBERHARDT, S.; HRICAK, H. y cols.: "Transition zone prostate cancer: metabolic characteristics at $\mathrm{H} 1 \mathrm{MR}$ spectroscopic Imaging-Initial results". Radiology, 229: 241, 2003.

**17. DJAVAN, B.; REMZI, M.; GHAWIDEL, K. y cols.: "Diagnosis of prostate cancer: the clinical use of transrectal ultrasound and biopsy". EAU Update Series, Vol. I: 9, 2003.

18. VILANOVA, J.C.; COMET, J.; BARCELÓ, J. y cols.: "The value of MR spectroscopic imaging for prostate cancer detection in the peripheral and transitional zone in patients with elevated PSA and negative biopsy". Comunicación. Pendiente publicar. 